\title{
Development and in vitro characterization of drug delivery system of rifapentine for osteoarticular tuberculosis
}

This article was published in the following Dove Press journal:

Drug Design, Development and Therapy

5 March 2015

Number of times this article has been viewed

\author{
Jun $\mathrm{Wu}^{\prime}$ \\ Yi Zuo ${ }^{2}$ \\ Yunjiu $\mathrm{Hu}^{\prime}$ \\ Jian Wang ${ }^{2}$ \\ Jidong $\mathrm{Li}^{2}$ \\ Bo Qiao' \\ Dianming Jiang' \\ 'Department of Orthopedics, The \\ First Affiliated Hospital of Chongqing \\ Medical University, Chongqing, \\ People's Republic of China; ${ }^{2}$ Research \\ Center for Nano-Biomaterials, \\ Analytical and Testing Center, \\ Sichuan University, Chengdu, Sichuan, \\ People's Republic of China
}

\begin{abstract}
The study was to develop and evaluate the rifapentine-loaded poly(lactic acid-co-glycolic acid) (PLGA) microspheres (RPMs) for the treatment of osteoarticular tuberculosis to avoid critical side effects caused by oral regimens of antibiotics or intravenous antibiotics. The RPMs were spherical with rough surfaces, and elevated amounts of rifapentine in the formulation markedly increased the particle size and drug loading, while decreased the size distribution and entrapment efficiency. The highest drug loading and encapsulation efficiency of RPMs were $23.93 \% \pm 3.93 \%$ and $88.49 \% \pm 8.49 \%$, respectively. After the initial rapid drug release, the release rate gradually decreased, and approximately $80 \%$ of the encapsulated rifapentine was released after 30 days of incubation. Moreover, RPMs could effectively inhibit the growth of Staphylococcus aureus. With increasing rifapentine content, the inhibition zones were continuously enlarged while the minimal inhibitory concentration values decreased. These results suggested that RPMs were bioactive and controlled release delivery systems for the treatment of osteoarticular tuberculosis.
\end{abstract}

Keywords: Staphylococcus aureus, antitubercular drugs, in vitro, PLGA microspheres, chemotherapy, antibacterial

\section{Introduction}

Osteoarticular tuberculosis, which accounts for approximately $10 \%-20 \%$ of all diagnosed tuberculosis, is the most common extrapulmonary tuberculosis. ${ }^{1}$ Antitubercular drugs have been used not only in the mild osteoarticular tuberculosis but also in the perioperation of infection foci debridement, remaining the cornerstone for the treatment of osteoarticular tuberculosis. At present, osteoarticular tuberculosis has been treated according to the standard therapy protocol by antitubercular drugs for a minimum of 8 and 20 months for total chemotherapy. ${ }^{2}$ However, longer treatment time resulted in patient noncompliance and critical side effects. ${ }^{3}$ It is key for the treatment of osteoarticular tuberculosis to increase local drug concentrations for higher therapeutic efficiencies and lower toxicity for body. Although antitubercular drugs implanted in the infection foci can achieve these requirements, they will be rapidly absorbed, possibly resulting in failure and recurrence. Research for more efficient therapeutic approaches for the treatment of tuberculosis has been carried out by using drug delivery systems. ${ }^{4-6}$ These systems are indeed expected to deliver medicines to the target tissue more specifically and release the drugs continually, enhancing the bioavailability and reducing the adverse effects. ${ }^{7}$ Among the various drug delivery systems, microspheres composed of biodegradable polymers, especially poly(lactic acid-co-glycolic acid) (PLGA) microspheres, have brought up tremendous interest. ${ }^{8,9}$
Correspondence: Dianming Jiang First Affiliated Hospital of Chongqing Medical University, No.I Youyi Road, Yuzhong District, Chongqing 400016, People's Republic of China

Tel +862389011212

Fax +862389011212

Email jdm571026@vip.163.com 
PLGA is approved by the US Food and Drug Administration (FDA) as a base for medical treatment due to its biocompatibility and biodegradability. PLGA microspheres have been successfully formulated to entrap the front-line antituberculosis agents, ${ }^{10}$ especially rifampicin-loaded PLGA microspheres. ${ }^{5,11}$ They have satisfactory characteristics and controlled release properties and exhibited great potential application in the field of tuberculosis treatment. However, to the best of our knowledge, the drug contents of most rifampicin-loaded PLGA microspheres are relatively low (from $4.9 \%$ to $16.5 \%){ }^{4}{ }^{4}$ therefore, a higher dosage is needed to achieve effective concentrations.

Rifapentine is a semisynthetic antibiotic belonging to the rifamycin group and has been approved to be one of the firstline medicines for the treatment of tuberculosis by the FDA. Compared with rifampicin, rifapentine has similar antibacterial spectrum, but approximately ten times greater antibacterial potency against Mycobacterium tuberculosis bacteria (MTB) in vitro, ${ }^{3}$ and milder adverse reaction. Furthermore, rifapentine possesses a very important feature: it can prevent drug resistance. ${ }^{12}$ Taking these conditions into account, rifapentine was chosen as a model drug to formulate PLGA microspheres, which have not been reported yet in the literature.

In this study, rifapentine-loaded PLGA microspheres (RPMs) were prepared by emulsion solvent evaporation method. The physiochemical properties of RPMs were characterized, including morphology, size distribution, and encapsulation efficiency. In vitro drug release studies were carried out in phosphate-buffered saline (PBS, $0.2 \mathrm{M}, \mathrm{pH} 7.4$ ) mediums at $37.1^{\circ} \mathrm{C}$. Furthermore, the in vitro antibacterial properties of the microspheres were detected by agar diffusion test (ADT) and minimal inhibitory concentration (MIC).

\section{Materials and methods Materials}

Poly(lactic-co-glycolic acid) (PLGA, lactic/glycolic $=50: 50)$ with an average molecular weight of 45,000 was purchased from Daigang Biomaterials Co. Ltd., Jinan, People's Republic of China. Rifapentine was purchased from Ruibio Ltd., Germany. The polyvinyl alcohol 1799 (PVA 1799, Sinopec Sichuan Vinylon Factory, Chongqing, People's Republic of China) was used as an emulsion stabilizer. Müeller-Hinton $(\mathrm{MH})$ broth and agar powder were purchased from Oxoid Ltd., Hampshire, UK. The strains of Staphylococcus aureus (S. aureus, ATCC 6538) were procured from the clinical laboratory of the first affiliated hospital of Chongqing Medical University. All other chemical reagents were of analytical grade.

\section{Microsphere preparation}

Since rifapentine is a hydrophobic compound, RPMs were prepared by oil-in-water $(\mathrm{O} / \mathrm{W})$ emulsion solvent evaporation method. ${ }^{13}$ Briefly, $200 \mathrm{mg}$ of PLGA was dissolved in $10 \mathrm{~mL}$ dichloromethane (DCM), and different amounts of rifapentine $(0,20,50,100 \mathrm{mg}$, named RPMs0, RPMs20, RPMs50, and RPMs100) were dissolved into the polymer solution. The polymer-solvent-drug solution (dispersed phase) was uniformly injected into $100 \mathrm{~mL}$ of $2 \mathrm{wt} \%$ PVA 1799 aqueous solution (continuous phase). The resulting mixture was emulsified by mechanical stirring (about $700 \times g$ ) for 30 minutes at room temperature to yield an $\mathrm{O} / \mathrm{W}$ emulsion. Subsequently, the emulsion was gently stirred (about $250 \times \mathrm{g}$ ) at room temperature until the DCM volatilized completely. The hardened microspheres were collected by centrifugation $(2,000 \times g$, 10 minutes) and washed three times with triple-distilled water. The microspheres were freeze dried overnight to obtain free-flowing particles and finally stored at $-20^{\circ} \mathrm{C}$.

\section{Morphologies}

The morphologies of RPMs were observed under a scanning electron microscope (SEM, JSM-6500LV, JEOL, Tokyo, Japan). The dry specimens were mounted onto sample stubs using double-sided adhesive tape and then coated with gold palladium under vacuum. The coated microspheres were observed under the SEM at $15 \mathrm{kV}$.

\section{Size distribution}

The size distribution of the microspheres was measured using laser particle size analyzer (JL-6000, Chengdu Jingxin Power Analysis Instruments Ltd., Chengdu, People's Republic of China), and the D50 volume diameter was taken as the mean diameter of the microspheres.

\section{Rifapentine content and encapsulation efficiencies}

To detect the drug content and encapsulation efficiency of the RPMs, 10 mg of RPMs was accurately weighed and dissolved in $10 \mathrm{~mL}$ of methanol. After incubation at $37.1^{\circ} \mathrm{C}$ overnight, the rifapentine in the solution was analyzed by UV-visible spectrophotometry (SP-754, Shanghai Spectrum, People's Republic of China) at a wavelength of $475 \mathrm{~nm}$. The drug contents in the RPMs were calculated with a calibration curve of rifapentine, which was achieved from rifapentine solution in methanol with a concentration between 0.03 and $27 \mathrm{mg} / \mathrm{L}$ $\left(R^{2}=0.9997\right)$. The encapsulation efficiency was defined as the percent ratio of the actual rifapentine loading to the theoretical rifapentine loading. Tests were done in triplicate. 


\section{In vitro rifapentine release}

The in vitro rifapentine release studies were carried out in PBS (0.2 M, pH 7.4). About $10 \mathrm{mg}$ of RPMs was enclosed in a dialysis bag with a molecular weight cutoff of 3,500 , and then placed in a $50 \mathrm{~mL}$ centrifuge tube which was prefilled with $10 \mathrm{~mL}$ PBS. The tube was placed in a shaking water bath $\left(37^{\circ} \mathrm{C}\right.$ and $\left.100 \times g\right)$, and the PBS was replenished every day. At appropriate time intervals, the supernatant of the PBS solution was collected and the optical density was read at a wavelength of $475 \mathrm{~nm}$ by UV-visible spectrophotometry. The release rates and cumulative release profiles were calculated and mapped. The analysis of each sample was repeated three times.

\section{In vitro antibacterial activity}

\section{Bacterial suspension}

Rifapentine is a broad-spectrum antibiotic and has a highly antibacterial activity toward most gram-positive cocci. Therefore, S. aureus (ATCC 6538) was used in this study to investigate the in vitro antibacterial performance of RPMs. A single colony of $S$. aureus was inoculated in $\mathrm{MH}$ broth and incubated at $37^{\circ} \mathrm{C}$ for 24 hours in an incubator (Mermmet model 800). Then the bacterial suspension was diluted with $\mathrm{MH}$ broth to $10^{6}$ colony-forming units per milliliter (CFU/mL).

\section{Agar diffusion test}

Susceptibility of $S$. aureus strains to RPMs was determined by the ADT with a filter paper. About $10 \mathrm{mg}$ of the samples were suspended in $10 \mathrm{~mL}$ of sterile PBS for 24 hours. The PBS suspensions were centrifuged $(1,000 \times g, 5$ minutes) to acquire supernatants, in which the sterile filter papers with a diameter of $10 \mathrm{~mm}$ were immersed. Thirty minutes later, the filter papers were withdrawn, the excess liquid was drained off, and then irradiated by ultraviolet radiation three times, every half an hour. About $50 \mu \mathrm{L}$ of bacterial suspensions were homogeneously spread on $\mathrm{MH}$ agar plates. The filter papers were carefully placed in the center of the plates with sterile tweezers. After incubation at $37^{\circ} \mathrm{C}$ for 20 hours, the agar plates were examined for the inhibition zone. The pure rifapentine and unloaded PLGA microspheres were used as positive and negative controls.

\section{Minimal inhibitory concentration}

The MIC values of rifapentine and RPMs were determined by the broth macrodilution method according to the standard M07-A9 recommended by the Clinical and Laboratory Standards Institute. ${ }^{14}$ About $12.8 \mathrm{mg}$ of the samples were dissolved in $5 \mathrm{~mL}$ of sterile $\mathrm{MH}$ broth and serially diluted to final concentrations of $0.3 \mu \mathrm{g} / \mathrm{mL}$ (rifapentine) and $5 \mu \mathrm{g} / \mathrm{mL}$ (RPMs). The freshly prepared bacteria suspension $(2 \mathrm{~mL}$; $10^{6} \mathrm{CFU} / \mathrm{mL}$ in $\mathrm{MH}$ broth) was added to the same volume of samples - MH broth. Therefore, the final concentration of the S. aureus was $5 \times 10^{5} \mathrm{CFU} / \mathrm{mL}$. Subsequently, the inoculated broth was incubated at $37^{\circ} \mathrm{C}$ for 16 hours. The MIC value was the lowest antibacterial agent concentration that can completely inhibit the growth of the bacterium as detected by the naked eye. Tests were performed in triplicate.

\section{Statistical analysis}

Results are expressed as means \pm standard deviation. Statistical analyses were performed using the SPSS 17.0 software. Comparisons between the groups were performed using one-way analysis of variance (ANOVA) with Tukey's test. A difference was considered statistically significant if the $P$-value was less than or equal to 0.05 .

\section{Results and discussion PLGA microspheres for rifapentine delivery}

The PLGA microspheres were well dispersed and mostly spherical in shape (Figure 1). The rifapentine-unloaded PLGA microspheres showed smooth surfaces without hollows or deformations (Figure 1A). Although the microspheres maintained their spherical shape, the rifapentine in the formulation significantly affected the surface morphology. The surfaces of the RPMs 20 were bumpy, since there were some shallow concaves (Figure 1B). As the initial drug-to-polymer ratio increased, the concaves on surfaces of the RPMs became deeper and changed into pores (Figure 1C and D). Meanwhile, the surface porosity increased significantly and the pores almost spread over the surfaces of the RPMs100 (Figure 1D). According to Gilchrist et al ${ }^{15}$ these concaves (pores) were formed due to the loss of solid-solid phase-separated domains of drug from the polymer during the microsphere hardening process.

Figure 2 presents the size distribution of three kinds of RPMs and one unloaded PLGA microspheres. The 50th volume particle diameters (D50) were $12.422 \mu \mathrm{m}$ for RPMs0, $16.737 \mu \mathrm{m}$ for RPMs20, $25.267 \mu \mathrm{m}$ for RPMs50, and $27.833 \mu \mathrm{m}$ for RPMs 100 . With the increase in the D50 of PLGA microspheres, the size distribution was narrowed gradually. Rifapentine is a hydrophobic chemical reagent that would increase the viscosity of the PLGA-DCM solution (the oil phase). Thus, because of the increasing amount of rifapentine in the formula, the oil phase was more difficult 
A

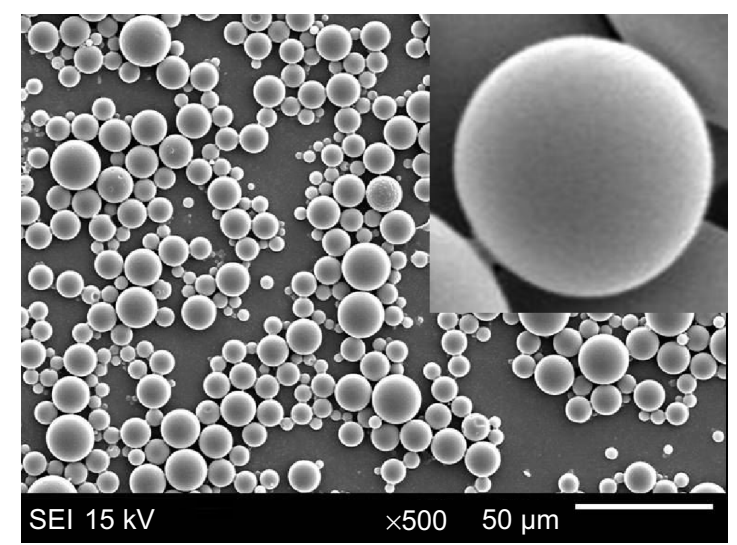

C

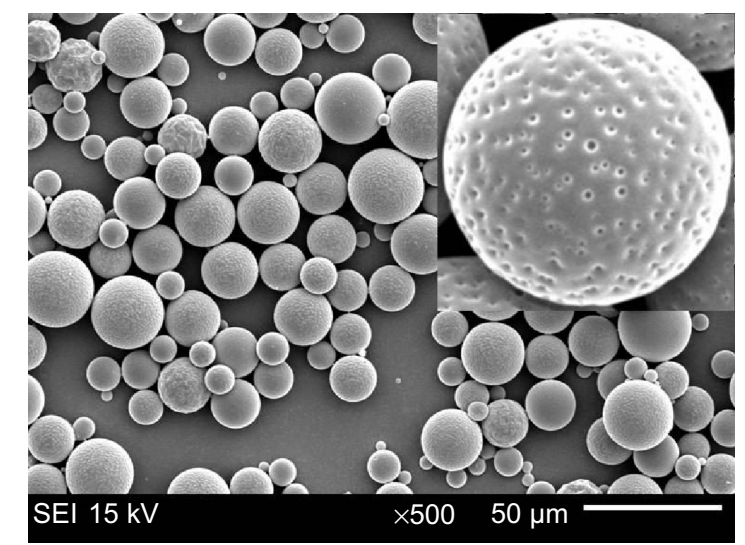

B
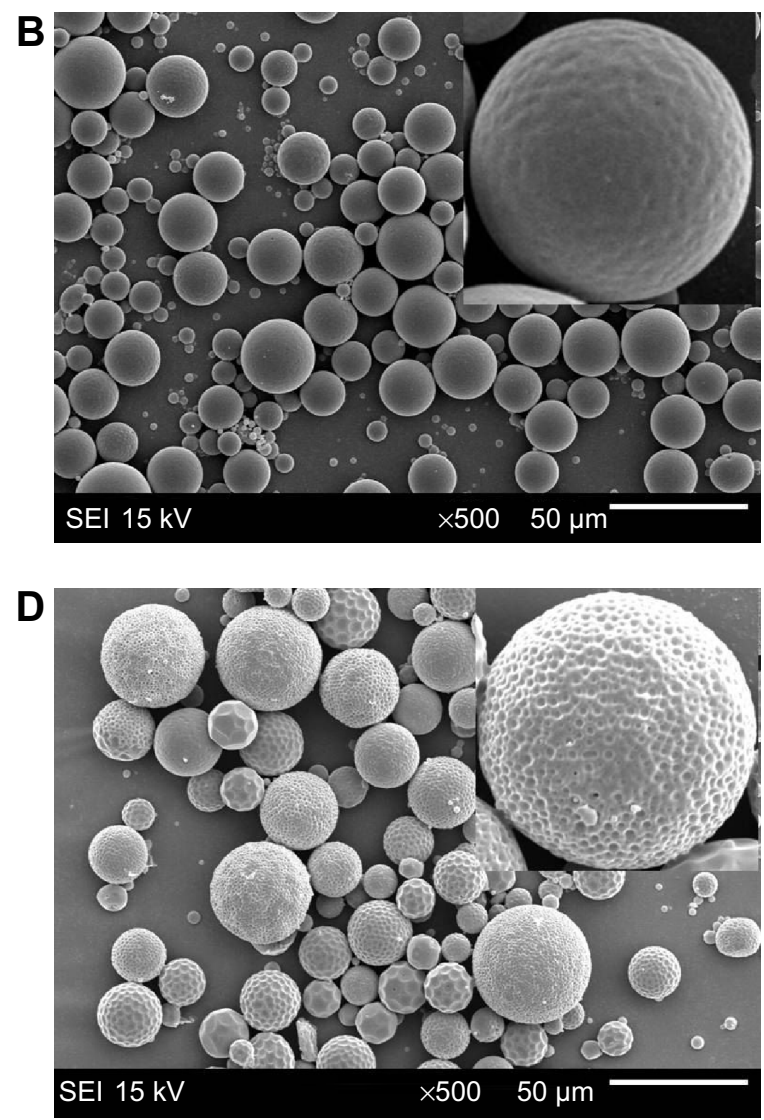

Figure I SEM images of unloaded PLGA microspheres and RPMs.

Notes: (A) RPMs0, (B) RPMs20, (C) RPMs50, (D) RPMs 100. RPMs0 denotes unloaded PLGA microspheres, RPMs20, RPMs50, and RPMs I00 denote rifapentine-loaded PLGA microspheres with 20,50 , and $100 \mathrm{mg}$ of rifapentine in formula, respectively.

Abbreviations: SEl, secondary electron image; SEM, scanning electron microscope; PLGA, poly(lactic acid-co-glycolic acid); RPMs, rifapentine-loaded PLGA microspheres.

to be dispersed into the external aqueous phase during the second emulsification process.

The drug loading and encapsulation efficiency of the RPMs are summarized in Table 1. It is obvious that, with an increase

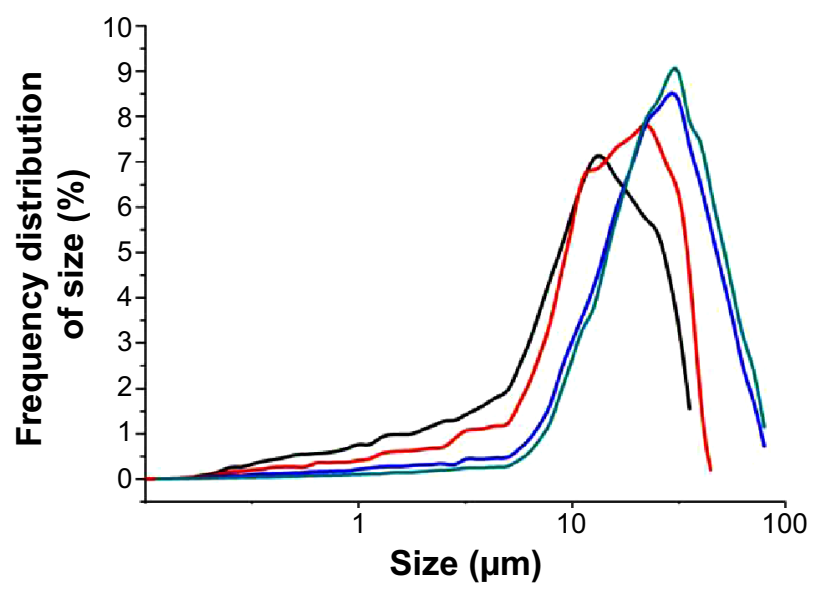

Figure 2 Size distribution of unloaded PLGA microspheres and RPMs.

Notes: Black line: RPMs0, red line: RPMs20, blue line: RPMs50, green line: RPMs 100 . RPMs0 denotes unloaded PLGA microspheres, RPMs20, RPMs50, and RPMs 100 denote rifapentine-loaded PLGA microspheres with 20,50, and $100 \mathrm{mg}$ of rifapentine in formula, respectively.

Abbreviations: PLGA, poly(lactic acid-co-glycolic acid); RPMs, rifapentine-loaded PLGA microspheres. of rifapentine theoretical drug loading, the actual drug loading increased, since there was more rifapentine to be encapsulated by the PLGA polymer matrix. However, the entrapment efficiency decreased with the increase of theoretical drug loading. We all know that fast polymer precipitation on the surface of the dispersed phase can prevent drug loss into the external continuous phase. ${ }^{16}$ Rifapentine delayed the solidification of the polymer-solvent-drug solution, and more rifapentine molecules were diffused into the continuous phase under the action of concentration potential energy. What's more, according to Gilchrist et $\mathrm{al}^{15}$ the lower encapsulation efficiency in the RPMs with higher rifapentine loadings might be explained by the loss of solid-solid phase-separated domains of drug from the polymer. As shown in Figure 1, elevated amounts of rifapentine in the formulation markedly increased the surface porosity, losing more phase-separated domains and reducing the encapsulation efficiency.

In view of the effects of rifapentine on the morphology, size distribution, drug loading, and encapsulation efficiency of the RPMs, the optimum parameters of RPMs preparation in our study were as follows: $50 \mathrm{mg}$ of rifapentine and $200 \mathrm{mg}$ of PLGA dissolved in $10 \mathrm{~mL}$ DCM, 
Table I Drug loading and entrapment efficiency of rifapentine-loaded PLGA microspheres

\begin{tabular}{llllll}
\hline Group & PLGA (mg) & Rifapentine $(\mathbf{m g})$ & Theoretical drug loading (\%) & Actual drug loading (\%) & Entrapment efficiency (\%) \\
\hline RPMs20 & 200 & 20 & 9.09 & $8.04 \pm 0.29$ & $88.49 \pm 3.23$ \\
RPMs50 & 200 & 50 & 20.00 & $17.16 \pm 0.40^{\mathrm{a}}$ & $85.78 \pm 2.00^{\mathrm{b}}$ \\
RPMs100 & 200 & 100 & 33.33 & $23.93 \pm 0.48^{\mathrm{a}, \mathrm{c}}$ & $71.80 \pm 1.45^{\mathrm{b}, \mathrm{d}}$ \\
\hline
\end{tabular}

Notes: $\mathrm{n}=3$, mean \pm SD. RPMs20, RPMs50, and RPMs 100 denote rifapentine-loaded PLGA microspheres with 20 , 50 , and I00 mg of rifapentine in formula, respectively. ${ }^{a, b}$ Compared with RPMs20, $P<0.05$. ${ }^{c, d}$ Compared with RPMs50, $P<0.05$.

Abbreviation: PLGA, poly(lactic acid-co-glycolic acid).

and then uniformly injected into $100 \mathrm{~mL}$ of $2 \mathrm{wt} \%$ PVA 1799 aqueous solution. The mean diameter of the RPMs50 was $25.267 \mu \mathrm{m}$, and the drug loading and encapsulation efficiency were $17.16 \% \pm 0.40 \%$ and $85.78 \% \pm 2.00 \%$, respectively. In 2011, Doan et $\mathrm{al}^{4}$ optimized the process parameters of solvent evaporation method to produce rifampicin-loaded PLGA microspheres with satisfactory rifampicin contents (from $4.9 \%$ to $16.5 \%$ ). The maximal drug loading of rifampicin-loaded PLGA microspheres prepared by Lawlor et $\mathrm{al}{ }^{17}$ using a solvent evaporation process was $25.67 \pm 0.73 \mu \mathrm{g}$ rifampicin per mg of PLGA, which represented an encapsulation efficiency of $16.75 \% \pm 0.49 \%$. It is obvious that the drug loading and encapsulation efficiency of the RPMs in this study were higher than those of the two rifampicin-loaded PLGA microspheres mentioned earlier. Since rifapentine had approximately ten times greater antibacterial potency against MTB in vitro, the antibacterial potency of RPMs was much higher than that of the rifampicin-loaded PLGA microspheres under the same dosage. Furthermore, the encapsulation efficiency was also higher than the highest encapsulation efficiency $(72.08 \% \pm 1.90 \%)$ of rifapentine-loaded proliposomes, prepared by single-step spray drying method ${ }^{18}$ It is well known that improving the encapsulation efficiency and drug loading could reduce not only the loss of the bioactive substances but also the administration dosage of microspheres.

\section{In vitro rifapentine release}

The release characteristic is an important aspect that must be examined during the research and development process of a drug delivery system. The in vitro release test was an in vitro evaluation method of microspheres, and the results of three types of RPMs are shown in Figures 3 and 4. All the three types of microspheres in our tests exhibited a similar biphasic release profile. A large amount of rifapentine was released during the first 2 days, which was followed by a period of decreased release rate for nearly 4 weeks. At the end of the in vitro release tests, the cumulative release percentages of rifapentine were $72.10 \% \pm 0.26 \%$ for RPMs $20,80.22 \% \pm 0.56 \%$ for RPMs 50 , and $78.59 \% \pm 0.14 \%$ for RPMs 100 .
The initial release increased with increasing dosage of rifapentine in the formulation, which was $9.07 \% \pm 0.11 \%$ for RPMs $20,13.33 \% \pm 0.38 \%$ for RPMs 50 , and $15.50 \% \pm 0.09 \%$ for RPMs 100 . The initial fast release was usually described as a burst release, which might be attributed to the surfacebound and poorly encapsulated drugs close to the surface. ${ }^{19}$ From the section PLGA Microspheres for Rifapentine Delivery in this paper, we know that a higher rifapentine dosage facilitates the formation of more holes, which enlarge the surface area per unit volume exposed to the release medium. The porous structure of the PLGA microspheres allowed the drug trapped in the layers near the surface to be rapidly released..$^{20}$ Furthermore, the higher the drug loading, the more drug molecules on the surface, which could contribute significantly to the burst effect. Therefore, the higher dosage of rifapentine in the formulation led to higher initial burst.

Subsequently, the release phase shifted to a sustained release one, during which rifapentine was released at a slow and gradually decreasing rate. According to Fredenberg et $\mathrm{a}^{21}$ the sustained release phase was often attributed to the combined effects of diffusion and erosion of the PLGA substrate. Rifapentine was released by diffusion through the water-filled

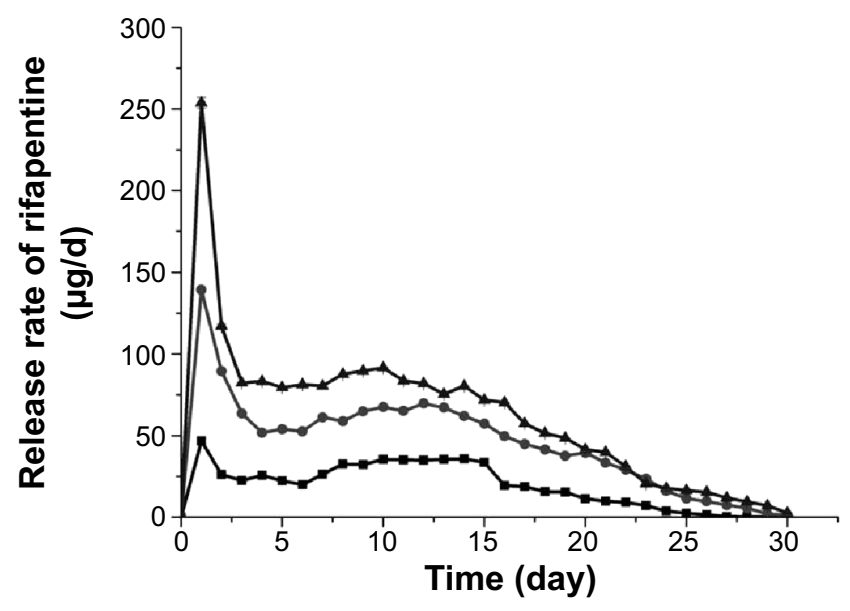

Figure 3 In vitro release rate of rifapentine from RPMs with different rifapentine content in PBS (0.2 M, pH 7.4).

Notes: Squares: RPMs20 (8.04\%); circles: RPMs50 (17.16\%); triangles: RPMs 100 (23.93\%). RPMs20, RPMs50, and RPMsI00 denote rifapentine-loaded PLGA microspheres with 20,50 , and $100 \mathrm{mg}$ of rifapentine in formula, respectively.

Abbreviations: RPMs, rifapentine-loaded PLGA microspheres; PLGA, poly(lactic acid-co-glycolic acid); PBS, phosphate-buffered saline. 


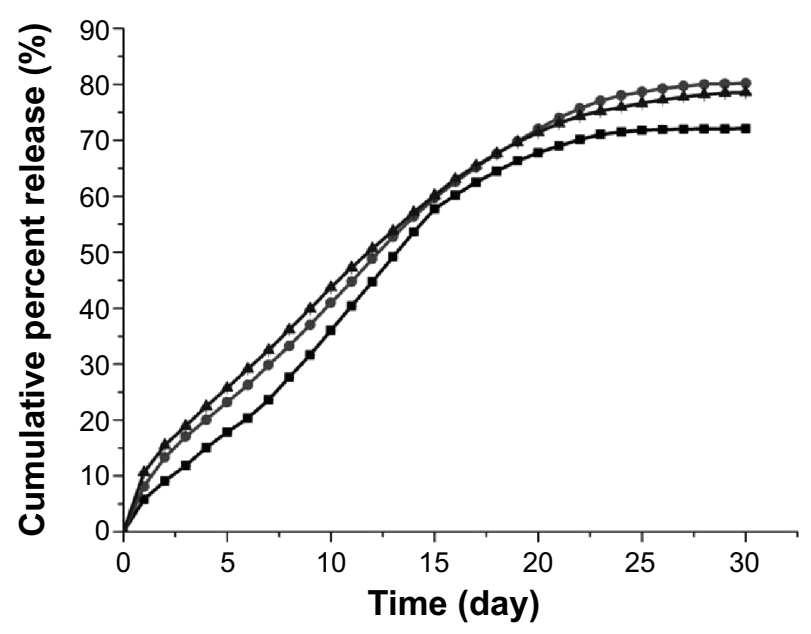

Figure 4 In vitro cumulative percent release of rifapentine from RPMs with different rifapentine content in PBS (0.2 M, pH 7.4).

Note: Squares: RPMs20 (8.04\%); circles: RPMs50 (17.16\%); triangles: RPMsI00 (23.93\%). RPMs20, RPMs50, and RPMsI00 denote rifapentine-loaded PLGA microspheres with 20,50 , and $100 \mathrm{mg}$ of rifapentine in formula, respectively. Abbreviations: RPMs, rifapentine-loaded PLGA microspheres; PLGA, poly(lactic acid-co-glycolic acid); PBS, phosphate-buffered saline.

pores which were formed by the inner aqueous phase during the fabrication process, diffusion of the drug, degradation, and subsequent erosion of the PLGA substrate. It could be observed in Figure 3 that, at the most time point, the higher the drug loading, the greater the rifapentine release. There are two possible explanations for this phenomenon. On the one hand, the diffusion might be promoted by the rifapentine concentration gradient between the RPMs and the dissolution medium. On the other, the porosity was increased by diffusion of the drug and erosion of the PLGA substrate, leading to more PLGA substrates contacting with the dissolution medium, facilitating further drug release and erosion of the PLGA substrate..$^{22,23}$

This kind of drug release characteristic possessed some clinical significance in the treatment of osteoarticular tuberculosis. During the period of the initially fast release phase, a large amount of rifapentine was released to obtain a high concentration level in the lesion site of infection, killing the pathogenic bacteria quickly and effectively. Subsequently, the slow and sustained drug release was conducive to maintain an effective drug concentration for a long time, to inhibit the growth of bacteria sustainably, and to prevent recrudescence.

\section{In vitro antibacterial activity}

The antibacterial activity of the RPMs against $S$. aureus was evaluated by the ADT and MIC. The results of ADT are shown in Figure 5. The pure PLGA microspheres, which were used as negative control, do not affect the bacterial growth. However, the pure rifapentine showed a markedly large inhibition zone $(21.88 \pm 0.40) \mu \mathrm{m}$, which was larger than $20 \mu \mathrm{m}$. According to the standards M100-S23 recommended by the Clinical and Laboratory Standards Institute, ${ }^{24}$ $S$. aureus strains used in our research are susceptible to rifapentine. Therefore, S. aureus strains were used to evaluate the antibacterial activities of rifapentine and RPMs. The average diameter of the inhibition zones were $7.62 \pm 1.02 \mu \mathrm{m}$ for RPMs20, $11.27 \pm 0.58 \mu \mathrm{m}$ for RPMs50, and 13.38 \pm 1.01 $\mu \mathrm{m}$ for RPMs100, showing that the increase of rifapentine loading enlarged the inhibition zones.

The MIC of rifapentine against $S$. aureus is shown in Figure 6. Under the naked eyes, the MH broth had already been cloudy since the tenth tube. Therefore, the concentration of rifapentine in the ninth tube $(5 \mu \mathrm{g} / \mathrm{mL})$ was the MIC value of rifapentine against $S$. aureus. Combined with the ADT results, a conclusion could be drawn that rifapentine possessed a high antimicrobial activity against $S$. aureus in vitro.

Since the MIC value of rifapentine was the concentration of rifapentine in the ninth tube, only nine concentration gradients of each kind of PLGA microspheres were used in our study. The results of the tests are plotted in Figure 7. The MIC values of RPMs against $S$. aureus were $40 \mu \mathrm{g} / \mathrm{mL}$ (RPMs 100 , Figure 7A), $80 \mu \mathrm{g} / \mathrm{mL}$ (RPMs50, Figure 7B), and $320 \mu \mathrm{g} / \mathrm{mL}$ (RPMs20, Figure 7C). The MIC values continuously decreased with the increase of rifapentine loading, and the results were in good agreement with those of ADT. At the same bacterial concentration, the inhibition zones and MIC values were determined by the amount of antimicrobial active substances in the medium. Thus, by combining these two assays, we were able

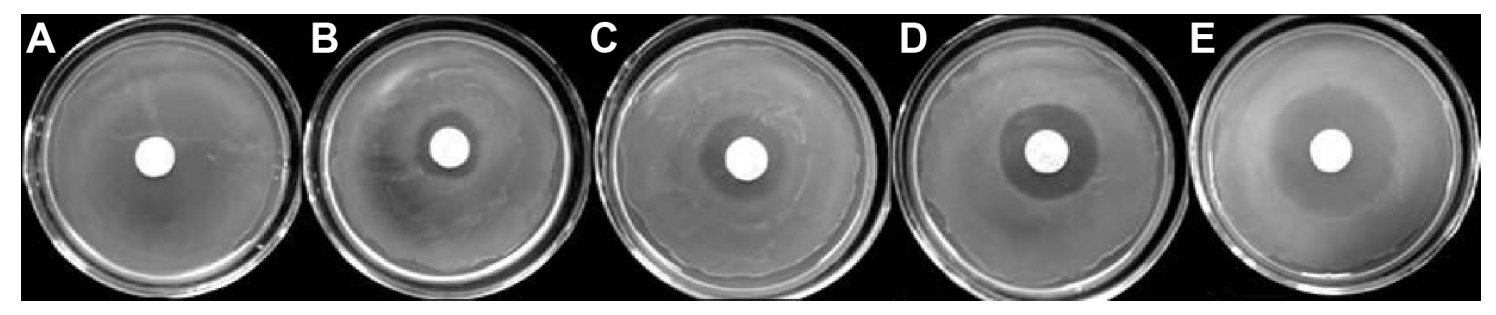

Figure 5 The agar diffusion tests of unloaded PLGA microspheres, RPMs, and rifapentine against Staphylococcus aureus strains.

Notes: (A) RPMs0, (B) RPMs20 (8.04\%), (C) RPMs50 (I7.16\%), (D) RPMs 100 (23.93\%), (E) rifapentine. RPMs0 denotes unloaded PLGA microspheres, RPMs20, RPMs50, and RPMs 100 denote Rifapentine-loaded PLGA microspheres with 20,50, and $100 \mathrm{mg}$ of rifapentine in formula, respectively.

Abbreviations: PLGA, poly(lactic acid-co-glycolic acid); RPMs, rifapentine-loaded PLGA microspheres. 


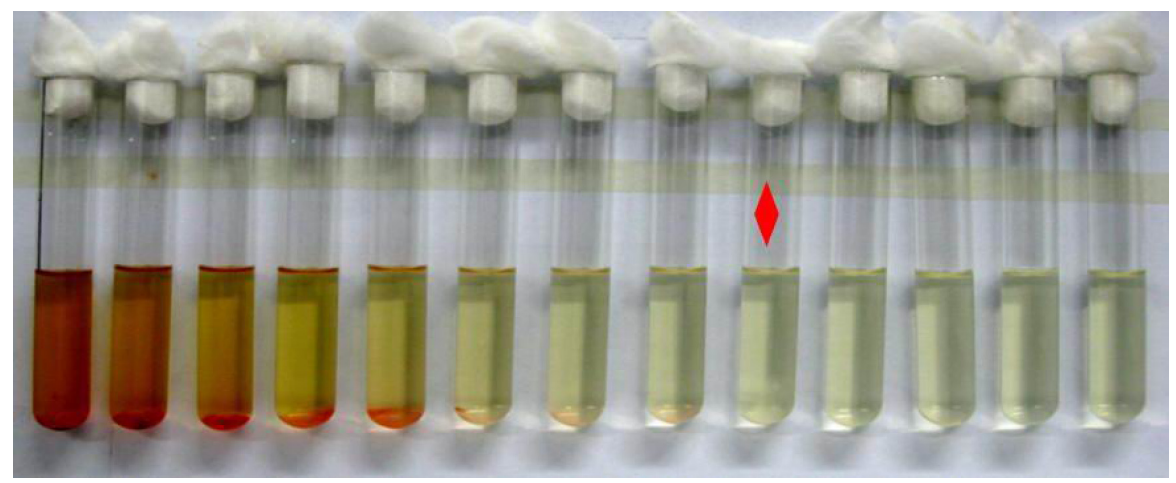

Figure 6 The MIC of rifapentine against Staphylococcus aureus strains.

Notes: Red diamond: the lowest antibacterial agent concentration that can completely inhibit the growth of the bacterium as detected by the naked eye.

Abbreviation: MIC, minimal inhibitory concentration.

to conclude that the antimicrobial potency of the RPMs was attributed to the release of rifapentine from the RPMs and its seepage into the medium. On increasing the rifapentine content of the RPMs, the concentration differences between the microspheres and the medium were increased and more rifapentine was released. Therefore, the inhibition zones enlarged while the MIC values decreased. In view of the antimicrobial activity of RPMs against $S$. aureus, we think that the rifapentine does not get inactivated during the preparation process (the emulsion solvent evaporation method).
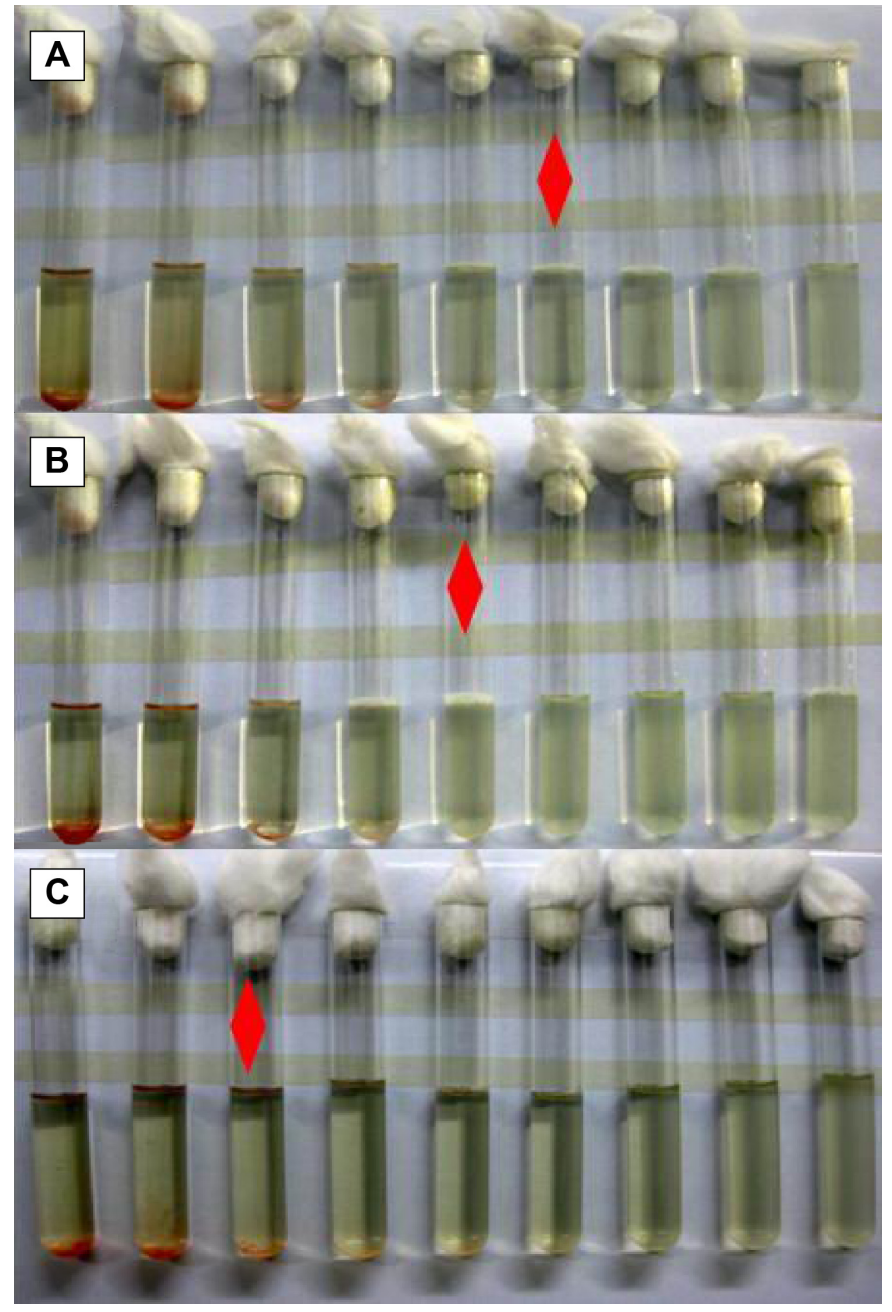

Figure 7 The MIC of RPMs with different rifapentine content against Staphylococcus aureus strains.

Notes: Red diamond: the lowest antibacterial agent concentration that can completely inhibit the growth of the bacterium as detected by the naked eye. (A) RPMs 100 (23.93\%), (B) RPMs50 (17.16\%), (C) RPMs20 (8.04\%). RPMs20, RPMs50, and RPMs 100 denote rifapentine-loaded PLGA microspheres with 20, 50 , and I00 mg of rifapentine in formula, respectively.

Abbreviations: MIC, minimal inhibitory concentration; RPMs, rifapentine-loaded PLGA microspheres; PLGA, poly(lactic acid-co-glycolic acid). 


\section{Conclusion}

In this study, rifapentine was successfully entrapped in PLGA microspheres using the $\mathrm{O} / \mathrm{W}$ emulsion solvent evaporation method. The optimum parameters of RPMs preparation in our study were $50 \mathrm{mg}$ of rifapentine and $200 \mathrm{mg}$ of PLGA dissolved in $10 \mathrm{~mL}$ DCM, and then uniformly injecting into $100 \mathrm{~mL}$ of $2 \mathrm{wt} \%$ PVA aqueous solution. Elevated amounts of rifapentine in the formulation markedly increased the particle size and drug loading, while decreasing the size distribution and entrapment efficiency of the RPMs. The highest drug loading and encapsulation efficiency of the RPMs were $23.93 \% \pm 0.48 \%$ and $88.49 \% \pm 3.23 \%$, respectively. After an initially rapid drug release during the first 2 days, the rifapentine release gradually slowed down and approximately $80 \%$ of the encapsulated rifapentine was released after 30 days of incubation. The in vitro antibacterial activity indicated that the RPMs were bioactive and able to kill the $S$. aureus in vitro. All these results suggest that the PLGA could be used as a good carrier for rifapentine to formulate a bioactive and controlled release delivery system that exhibits a great potential in the treatment of osteoarticular tuberculosis. Further research should be carried out to evaluate the biocompatibility and bioactivity of the RPMs in vivo.

\section{Acknowledgment}

This study was supported by the National Natural Science Foundation of China (Grant No 81171685).

\section{Disclosure}

The authors report no conflicts of interest in this work.

\section{References}

1. Muradali D, Gold WL, Vellend H, et al. Multifocal osteoarticular tuberculosis: report of four cases and review of management. Clin Infect Dis. 1993; 17:204-205.

2. Suárez-García I, Noguerado A. Drug treatment of multidrug-resistant osteoarticular tuberculosis: a systematic literature review. Int $J$ Infect Dis. 2012;16:e774-e778.

3. Aristoff PA, Garcia GA, Kirchhoff PD, et al. Rifamycins - obstacles and opportunities. Tuberculosis. 2010;90:94-118.

4. Doan T, Couet W, Olivier J. Formulation and in vitro characterization of inhalable rifampicin-loaded PLGA microspheres for sustained lung delivery. Int J Pharm. 2011;414:112-117.
5. Hirota K, Hasegawa T, Nakajima T, et al. Delivery of rifampicin-PLGA microspheres into alveolar macrophages is promising for treatment of tuberculosis. J Control Release. 2010;142:339-346.

6. Kyung HH, Seok HW, Tae JK. In vitro degradation and drug-release behavior of electrospun, fibrous webs of poly(lactic-co-glycolic acid). J Appl Polym Sci. 2012;124:209-214.

7. Kaparissides C, Alexandridou S, Kotti K, et al. Recent advances in novel drug delivery systems. J Nanotechnol Online. 2006;2:1-11.

8. Danhier F, Ansorena E, Silva JM, et al. PLGA-based nanoparticles: an overview of biomedical applications. J Control Release. 2012;161:505-522.

9. Makadia HK, Siegel SJ. Poly lactic-co-glycolic acid (PLGA) as biodegradable controlled drug delivery carrier. Polymers. 2011;3:1377-1397.

10. Ain Q, Sharma S, Garg S, et al. Role of poly [DL-lactide-co-glycolide] in development of a sustained oral delivery system for antitubercular drug (s). Int J Pharm. 2002;239:37-46.

11. Hirota K, Hasegawa T, Nakajima T, et al. Phagostimulatory effect of uptake of PLGA microspheres loaded with rifampicin on alveolar macrophages. Colloid Surface B. 2011;87:293-298.

12. Chan JG, Bai X, Traini D. An update on the use of rifapentine for tuberculosis therapy. Expert Opin Drug Deliv. 2014;11:421-431.

13. Zhang YH, Wang Y, Kong XC, et al. Facile preparation of polylactic acid/ketoconazole composite microspheres by oil/water solvent evaporation method. Adv Mater Res. 2013;652:335-338.

14. Clinical and Laboratory Standards Institute. Methods for Dilution Antimicrobial Susceptibility Tests for Bacteria That Grow Aerobically; Approved Standard-Ninth Edition. Wayne, PA: Clinical and Laboratory Standards Institute; 2012.

15. Gilchrist SE, Rickard DL, Letchford K, et al. Phase separation behavior of fusidic acid and rifampicin in PLGA microspheres. Mol Pharm. 2012;9:1489-1501.

16. Yeo Y, Park K. Control of encapsulation efficiency and initial burst in polymeric microparticle systems. Arch Pharm Res. 2004;27:1-12.

17. Lawlor C, O'Sullivan MP, Rice B, et al. Therapeutic aerosol bioengineering of targeted, inhalable microparticle formulations to treat Mycobacterium tuberculosis (MTb). J Mater Sci Mater Med. 2012;23:89-98.

18. Patil-Gadhe A, Pokharkar V. Single step spray drying method to develop proliposomes for inhalation: a systematic study based on quality by design approach. Pulm Pharmacol Ther. 2014;27(2):197-207.

19. Janoria KG, Mitra AK. Effect of lactide/glycolide ratio on the in vitro release of ganciclovir and its lipophilic prodrug (GCV-monobutyrate) from PLGA microspheres. Int J Pharm. 2007;338:133-141.

20. Hsin MW, Jian JW, Chi-Hwa W. In vitro sustained release of human immunoglobulin $\mathrm{G}$ from biodegradable microspheres. Ind Eng Chem Res. 2001;40:933-948.

21. Fredenberg S, Wahlgren M, Reslow M, et al. The mechanisms of drug release in poly (lactic-co-glycolic acid)-based drug delivery systems - a review. Int J Pharm. 2011;415:34-52.

22. Batycky RP, Hanes J, Langer R, et al. A theoretical model of erosion and macromolecular drug release from biodegrading microspheres. J Pharm Sci. 1997;86:1464-1477.

23. Perugini $\mathrm{P}$, Genta I, Conti B, et al. Long-term release of clodronate from biodegradable microspheres. AAPS Pharm Sci Tech. 2001;2:6-14.

24. Clinical and Laboratory Standards Institute. Performance Standards for Antimicrobial Susceptibility Testing; Twenty-Third Informational Supplement. Wayne, PA: Clinical and Laboratory Standards Institute; 2013.

\section{Publish your work in this journal}

Drug Design, Development and Therapy is an international, peerreviewed open-access journal that spans the spectrum of drug design and development through to clinical applications. Clinical outcomes, patient safety, and programs for the development and effective, safe, and sustained use of medicines are a feature of the journal, which

\section{Dovepress}

has also been accepted for indexing on PubMed Central. The manuscript management system is completely online and includes a very quick and fair peer-review system, which is all easy to use. Visit http://www.dovepress.com/testimonials.php to read real quotes from published authors. 